\title{
Polymeric retinoid prodrug PG-4HPR enhances the radiation response of lung cancer
}

\author{
GUANGYING ZHU ${ }^{1,3}$, XIANYI CAO ${ }^{1}$, JOE Y. CHANG ${ }^{2}$, LUKA MILAS ${ }^{2}$, \\ SIDNEY WALLACE ${ }^{1}$ and $\mathrm{CHUN} \mathrm{LI}^{1}$ \\ ${ }^{1}$ Division of Radiation Oncology and ${ }^{2}$ Department of Experimental Diagnostic Imaging, The University of \\ Texas M.D. Anderson Cancer Center, Houston, TX, USA; ${ }^{3}$ Department of Radiation Oncology, Peking University \\ School of Oncology, Beijing Cancer Hospital, Beijing Institution for Cancer Research, Beijing, P.R. China
}

Received March 22, 2007; Accepted May 18, 2007

\begin{abstract}
To determine whether a polymer-drug conjugate might improve tumor cell kill compared to the corresponding unconjugated agent when used in combination with radiation, we examined the antitumor activity of a water-soluble conjugate of $\mathrm{N}$-(4-hydroxyphenyl) retinamide (4HPR) and poly(L-glutamic acid), PG-4HPR, in lung cancer cells and xenografts. The antiproliferative activity of 4HPR and PG4HPR in human lung cancer A549 cells was evaluated and the response of the cells to radiation measured by clonogenic assay. Response to irradiation was evaluated by measuring tumor growth delay in nude mice bearing intramuscularly inoculated A549 tumors. Histologic responses were assessed by examination of apoptosis (TUNEL assay) and cell proliferation (Ki67 staining). In vitro, 4HPR and PG-4HPR inhibited the proliferation of A549 cells, with $\mathrm{IC}_{50}$ values of $25.8 \mu \mathrm{M}$ and $>50 \mu \mathrm{M}$, respectively, after $24 \mathrm{~h}$ of continuous exposure and $6.25 \mu \mathrm{M}$ and $9.75 \mu \mathrm{M}$, respectively, after $120 \mathrm{~h}$. Both agents increased radiosensitivity at an equivalent 4HPR concentration of $10 \mu \mathrm{M}$ after 5 days of exposure, with enhancement factors of 1.40 and 1.43. In tumor xenografts, intravenous injection of $4 \mathrm{HPR}$ or PG-4HPR $(30 \mathrm{mg}$ eq. $4 \mathrm{HPR} / \mathrm{kg}$ ) enhanced radiosensitivity by 1.3 and 1.6 , respectively, without apparent systemic toxicity. PG-4HPR augmented radiation-induced apoptosis and decreased cellular proliferation in vivo. The radiation response of A549 tumors was greater with PG-4HPR than with 4HPR, which may be attributed to increased delivery of 4HPR to the tumors and enhanced apoptotic response. These results suggest that a polymeric delivery system may be useful for modulating radiosensitivity.
\end{abstract}

Correspondence to: Dr Chun Li, Department of Experimental Diagnostic Imaging-Unit 59, The University of Texas M.D. Anderson Cancer Center, Houston, TX 77030, USA

E-mail: cli@di.mdacc.tmc.edu

Key words: radiosensitization, drug delivery, 4-HPR, poly(Lglutamic acid)

\section{Introduction}

It has been shown that the addition of concurrent chemotherapy to radiotherapy (chemoradiotherapy) results in improved clinical outcome in terms of both locoregional control and overall survival in some groups of patients (1-3). However, chemoradiotherapy is often associated with severe, dose-limiting toxicities that limit its application. For example, Liao et al (4) found that the combination of twice-daily radiotherapy (XRT) and concurrent cisplatin/etoposide resulted in a favorable long-term outcome (26\%, 5-year survival rate), but it also increased the incidence of more severe radiationinduced pneumonitis and esophagitis. Furthermore, most chemoradiotherapy regimens are platinum-based and there is evidence that the maximum tolerable toxicity has been reached with the dose intensities currently used in bolus cisplatin regimens. Therefore, new means are being explored to selectively target tumors with chemotherapeutic agents to reduce normal tissue toxicity and maximize antitumor efficacy.

One approach to improve the efficacy of radiotherapy without increasing its side effects is to use molecularly targeted therapeutic agents such as epidermal growth factor receptor and vascular endothelial growth factor receptor inhibitors $(1,5)$. The fact that the specificity of such agents will be high by design has given rise to the expectation that targeted drugs will be very active but generally well-tolerated. Alternatively, the novel drug delivery system may be used to achieve a high degree of spatial localization of chemotherapy within the tumor, which not only contributes to the efficacy of the drug as a pure chemotherapeutic agent, but also acts as a novel method for delivering chemotherapy as a radiosensitizer. It is known that long-circulating water-soluble polymers and nanoparticles exhibit enhanced tumor uptake after systemic administration. The phenomenon, termed enhanced permeability and retention (EPR) effect, is attributed to greater permeability of disordered capillary endothelia in malignant tumors towards macromolecules than normal tissue and the lack of functional lymphatics in solid tumors (6). Using paclitaxel as a model chemotherapeutic agent, we have previously demonstrated that combining radiotherapy and chemotherapy using a polymer-drug conjugate can lead to a stronger radiosensitizing effect than using the drug alone. Moreover, tumor irradiation can in turn potentiate the tumor 
response to polymer-drug conjugates by increasing tumor vascular permeability and thus the uptake of these conjugates into solid tumors (7-10). These intriguing preclinical data suggest a large potential benefit in using polymer-drug conjugates in combination with radiotherapy.

Promising low-toxicity agents for sensitizing tumors to radiotherapy or chemoradiotherapy include vitamin A and its synthetic analogues. Vitamin A (retinol) is a naturally occurring compound that plays a crucial role in the regulation of cellular differentiation and proliferation of epithelial tissue $(11,12)$. Retinoids include active metabolites of vitamin $\mathrm{A}$ as well as a diverse array of synthetic derivatives. Of the synthetic analogues, $N$-(4-hydroxyphenyl) retinamide (4HPR, fenretinide) has emerged as one of the most promising alternatives to the natural retinoids. 4HPR exhibits cytotoxic activity both in vitro and in vivo in a wide variety of tumors, including lung cancer cells (13-16). Multiple in vitro studies have demonstrated that retinoids can enhance the ability of radiation to kill cells and induce apoptosis (17-19). Thus far, however, no study has evaluated the radiosensitizing effect of 4HPR in an experimental animal model.

In this study, we have extended the use of a water-soluble polymeric drug conjugate as a radiosensitizer and investigated the antitumor effect of PG-4HPR in combination with radiation therapy in a human lung tumor model.

\section{Materials and methods}

Materials. 4HPR, PG $\left(\mathrm{M}_{\mathrm{n}}=13,000 \mathrm{Da}\right), 1,6$-hexandiol, dicyclohexyl carbodiimide, pyridine, diaminobenzidine, peroxide, and all solvents were purchased from SigmaAldrich (St. Louis, MO). Spectra/Pro 7 dialysis tubing (molecular weight cut-off, $10 \mathrm{kDa}$ ) was purchased from Fisher Scientific (Pittsburgh, PA). Silica gel plates for thinlayer chromatography were purchased from EM Science (Gibbstown, NJ).

Analytical methods. Ultraviolet (UV) measurements were recorded using a Beckman DU640 spectrophotometer (Beckman Coulter, Inc., Fullerton, CA). The concentration of $4 \mathrm{HPR}$ in polymer conjugate was quantified by UV measurement at $363 \mathrm{~nm}$. Gel permeation chromatography (GPC) was performed on a Waters high-performance liquid chromatography system (Waters Corp., Milford, MA) consisting of a 600 controller, a 717 plus auto sampler, and a

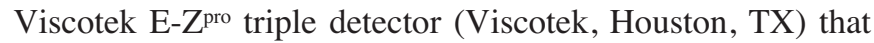
records refractive index, viscosity, and light-scattering signals. The samples were separated using a TSK-G4000PW $4.6 \mathrm{~mm}$ x $30 \mathrm{~cm}$ column (TosoHaas, Montgomeryville, PA) eluted with phosphate-buffered saline (PBS) containing $0.1 \% \mathrm{LiBr}$ at a flow rate of $1.0 \mathrm{ml} / \mathrm{min}$. Weight- and number-average molecular weight was calculated using Viscotek TriSEC GPC software.

Synthesis and characterization of $P G-4 H P R$. We used partially cross-linked PG to prepare PG-4HPR conjugate (Fig. 1). Partially cross-linked PG was prepared in situ from lowmolecular-weight linear PG. In a typical reaction, PG $\left(\mathrm{M}_{\mathrm{n}}\right.$, 13,000 Da, $2.0 \mathrm{~g}, 15.5 \mathrm{mmol} \mathrm{COOH})$ was dissolved in $120 \mathrm{ml}$ of anhydrous dimethylformaide, followed by the sequential

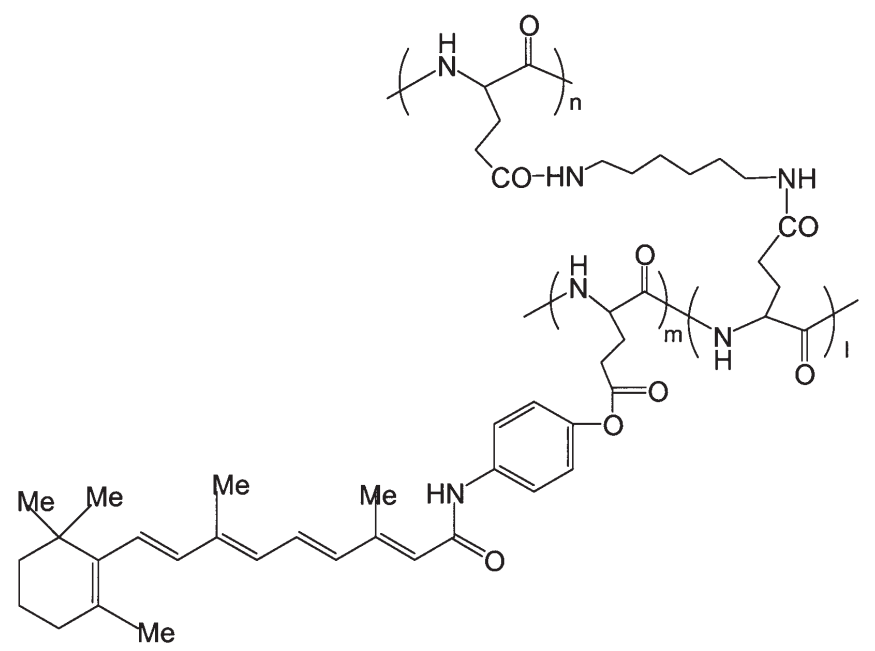

Figure 1. Structure of PG-4HPR synthesized from partially cross-linked PG.

addition of 1,6-hexandiol (131 mg, $1.1 \mathrm{mmol})$, pyridine (22 ml), and dicyclohexyl carbodiimide (800 mg, $3.88 \mathrm{mmol}$ ). The solution was stirred at room temperature for $24 \mathrm{~h}$. Next, 4HPR (0.2 g, $0.51 \mathrm{mmol})$ and dicyclohexyl carbodiimide (1000 mg, $4.85 \mathrm{mmol}$ ) were added to the above solution, and the resulting solution was stirred at room temperature for an additional $24 \mathrm{~h}$. After the removal of dicyclohexylurea by centrifugation and filtration, the product was precipitated from dimethylforamide (DMF) with chloroform. The precipitate was redissolved in $1 \mathrm{~N} \mathrm{NaCO}_{3}$ and dialyzed against distilled water (molecular weight cut-off, $10 \mathrm{~K}$ ) at $4^{\circ} \mathrm{C}$ with at least four exchanges. The aqueous solution was filtered through a $0.2-\mu \mathrm{m}$ membrane and lyophilized to yield $2.14 \mathrm{~g}$ of spongelike yellowish powder. The weight-average and numberaverage molecular weights of PG-4HPR conjugate were 86.5 K and 33.2 K, respectively. 4HPR content in PG-4HPR was $8.9 \%$ (yield: $95 \%$ ). Thin-layer chromatography confirmed the absence of free 4 HPR in the product.

Cell line and cell culture. The non-small cell lung cancer cell line A549 was obtained from American Type Culture Collection (Rockville, MD). Tumor cells were grown in F-12 medium supplemented with $10 \%$ fetal bovine serum (FCS, Life Technologies Europe, Paisley, UK) and $2 \mathrm{mM}$ glutamic acid and incubated at $37^{\circ} \mathrm{C}$ in a humidified $5 \% \mathrm{CO}_{2}$ atmosphere.

Growth inhibition. Stock solutions of 4HPR in dimethyl sulfoxide (DMSO, $10 \mathrm{mM}$ ) and PG-4HPR in water (equivalent $4 \mathrm{HPR}$ concentration, $10 \mathrm{mM}$ ) were prepared prior to the experiments. Cells were plated in 96 -well plates $\left(3 \times 10^{3}\right.$ cells/ well) and were allowed to grow at $37^{\circ} \mathrm{C}$ for $24 \mathrm{~h}$. The cells were then treated with $4 \mathrm{HPR}$ or PG-4HPR at various concentrations ranging from 0.08 to $50 \mu \mathrm{M}$ for 24,72 , and $120 \mathrm{~h}$. DMSO at a concentration of $0.5 \%(\mathrm{v} / \mathrm{v})$, equivalent to that used in $50 \mu \mathrm{M}$ of $4 \mathrm{HPR}$, was used as a control. Cell viability was measured using 3-(4,5-methylthiazol-2-yl)-2,5-diphenyltetrazolium bromide (MTT) assay. The absorbance was measured in triplicate at a wavelength of $570 \mathrm{~nm}$. The relative percentage of viable cells was calculated in terms of the 


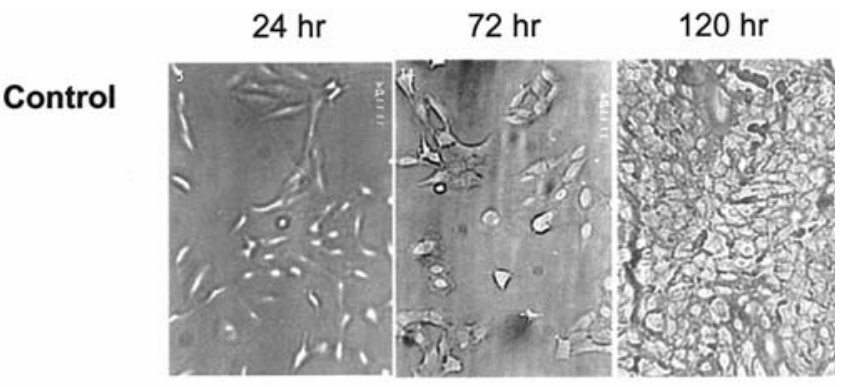

4HPR

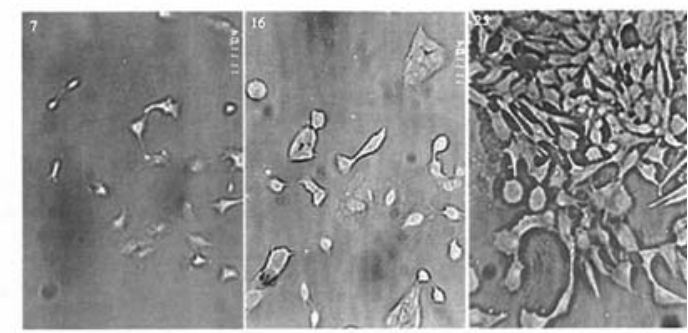

PG-4HPR

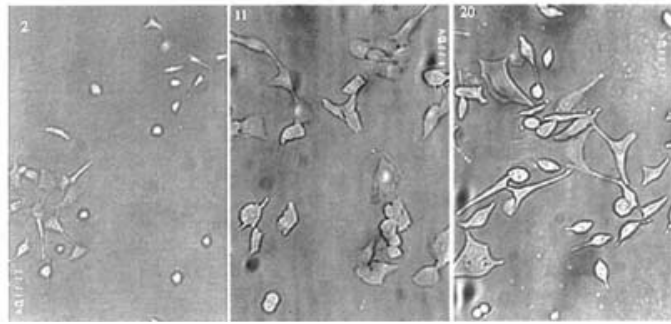

Figure 2. Morphologic changes of A549 cells induced by 4HPR and PG4HPR. Cells were treated with either 4HPR or PG-4HPR at a concentration of $50 \mu \mathrm{M}$ equivalent $4 \mathrm{HPR}$. Photographs show representative images from the control and treatment groups at different time points. PG-4HPR exhibited greater anti-proliferation activity than 4HPR did after prolonged exposure $(120 \mathrm{hr})$.

absorbance of treated cells compared with the absorbance of untreated control cells.

Radiosensitivity by clonogenic survival. A549 cells were cultured as described before. Twenty-four hours after cells were plated, the culture was replaced with medium containing $10 \mu \mathrm{M} 4 \mathrm{HPR}$ or PG-4HPR. This dose was chosen on the basis of the cell growth inhibition data, which showed that $\sim 50 \%$ of cell growth was inhibited at $\sim 10 \mu \mathrm{M}$ after treatment with $4 \mathrm{HPR}$ or PG-4HPR for 5 days. After continuous exposure for 5 days, the cells were irradiated with $0,1,2,4$, or 6 Gy of $\gamma$-rays using a ${ }^{137} \mathrm{Cs}$ source at a dose rate of $3.7 \mathrm{~Gy} / \mathrm{min}$. After irradiation, cells were counted and then plated in new dishes with medium containing $10 \mu \mathrm{M} 4 \mathrm{HPR}$ or PG-4HPR. At each radiation dose level, increasing numbers of cells (from 200 to 6000 cells per dish) were seeded. Thirteen days later, cell colonies were stained with crystal violet, and colonies over 50 cells were scored. Survival data were obtained for cells incubated with or without drugs.

Tumor growth in nude mice. Animals used in this study were maintained in facilities approved by the American Association for Accreditation of Laboratory Animal Care, and all experiments were performed in accordance with National Institutes of Health and institutional guidelines. Female nu/nu mice (4-6 weeks old) were purchased from Charles
River Laboratories (Wilmington, MA). The mice were maintained in a specific pathogen-free mouse colony.

Solitary tumors were produced by inoculation of $5 \times 10^{6}$ A549 cells into the muscle of the right hind legs of the mice. When the tumors had grown to $7-8 \mathrm{~mm}$ in average diameter, the mice were randomly divided into 6 groups of 6 mice each. Groups of tumor-bearing mice were treated as: 1) intravenous injection of $4 \mathrm{HPR}$ at a dose of $30 \mathrm{mg} / \mathrm{kg}, 2$ ) intravenous injection of PG-4HPR at a dose equivalent to $30 \mathrm{mg} 4 \mathrm{HPR} / \mathrm{kg}$, 3) local tumor irradiation alone, 4) 4HPR (30 mg/kg, i.v.) $24 \mathrm{~h}$ before local tumor irradiation, and 5) PG-4HPR (30 mg eq. 4HPR/kg, i.v.) $24 \mathrm{~h}$ before local tumor irradiation. Untreated mice served as controls. Before irradiation, mice were immobilized in a special jig, and tumors were centered in a 3-cm-diameter circular field. A single 10-Gy dose of gamma radiation was locally delivered using a dual-source ${ }^{137} \mathrm{Cs}$ unit at a dose rate of $6.25 \mathrm{~Gy} / \mathrm{min}$.

The effect of each treatment on tumor response was assessed by tumor growth delay. Three orthogonal tumor diameters were measured using calipers at 1-day intervals until the tumors grew to at least $14 \mathrm{~mm}$ in mean diameter. The degree of growth delay was expressed either as the absolute or normalized growth delay. Absolute growth delay was defined as the time in days for tumors in the treatment arms to grow from $7.5 \mathrm{~mm}$ to $12.5 \mathrm{~mm}$ in diameter minus the time in days for the tumors in the untreated control group to reach the same size. Normalized growth delay was defined as the time in days for tumors treated with a combined regimen to grow from $7.5 \mathrm{~mm}$ to $12.5 \mathrm{~mm}$ minus the time to reach the same size in tumors treated with 4HPR or PG-4HPR alone.

Histology. In a separate experiment, group of mice $(n=2)$ were treated as before and the mice were sacrificed by $\mathrm{CO}_{2}$ inhalation at $24 \mathrm{~h}$ after local irradiation. The tumors were immediately excised. Half of the tumor was stored in neutralbuffered formalin for hematoxilin and eosin (H\&E) staining. The other half was put on a plastic cassette, embedded in Tek Oct compound (Miles Scientific, Naperville, IL), frozen with liquid nitrogen, and then stored at $-160^{\circ} \mathrm{C}$. For H\&E staining, tissues were embedded in paraffin blocks, and $4-\mu \mathrm{m}$ sections were cut and stained with $\mathrm{H} \& \mathrm{E}$.

For terminal deoxyribonucleotidyl transferase-mediated nick end-labeling (TUNEL) and Ki67 staining, frozen tumor tissues were sectioned into $8-\mu \mathrm{m}$ slices. The DeadEnd ${ }^{\mathrm{TM}}$ colorimetric TUNEL system (Promega, Madison, WI) was used to stain for apoptotic cells. Frozen sections were washed by immersion in $0.85 \% \mathrm{NaCl}$ and $\mathrm{PBS}$ for 5 min each. The slides were then fixed in $10 \%$ buffered formalin in PBS for $15 \mathrm{~min}$ and washed with PBS twice for $5 \mathrm{~min}$ each. For cell permealization, slides were incubated with proteinase $\mathrm{K}$ solution (Amresco, Solon, $\mathrm{OH})(100 \mu 1,20 \mu \mathrm{g} / \mathrm{ml})$ for $15 \mathrm{~min}$. After a 5-min wash in PBS, the slides were fixed again in $10 \%$ buffered formalin in PBS for 5 min. Equilibration was then performed by incubation in $100 \mu$ l equilibration buffer for $5 \mathrm{~min}$ followed by another PBS wash. Labeling was preformed by adding $100 \mu 1$ of biotinylated terminal deoxynucleotidyl transferase (TdT) reaction mixture to the tissue sections on the slides. Plastic coverslips were used to ensure even distribution of TdT, and the slides were incubated for $60 \mathrm{~min}$ in a $37^{\circ} \mathrm{C}$ humidified chamber. The reaction was 


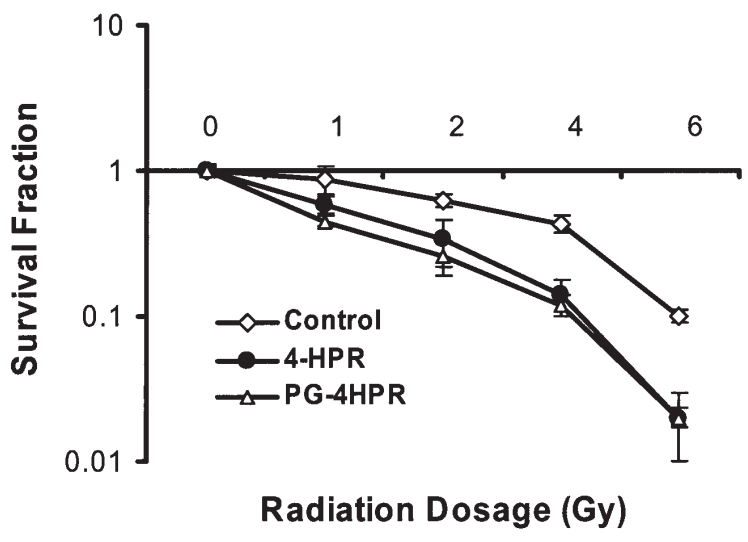

Figure 3. Enhancement of radiation response with $4 \mathrm{HPR}$ and PG-4HPR at an equivalent 4HPR concentration of $10 \mu \mathrm{M}$ in human lung cancer A549 cells. Untreated control or drug-treated A549 cells were irradiated at 0,1 , 2,4 , and 6 Gy. Cell colonies were counted after a 13-day incubation. Data represent mean values from 3 independent experiments. Bars $=$ standard deviation.

stopped by immersing the slides in $2 \mathrm{X}$ standard saline citrate for $15 \mathrm{~min}$, followed by three consecutive 5-min PBS washes. Immersion in $3 \%$ hydrogen peroxide for $3 \mathrm{~min}$ was done as the blocking step, followed by another triple wash in PBS. Streptavidin-horseradish peroxidase conjugate (100 $\mu 1$, diluted 1:500 in PBS) was then added, followed by 30 min of incubation. A triple wash in PBS was then preformed, followed by the addition of $100 \mu 1$ diaminobenzidine (Sigma-Aldrich), which was allowed to incubate until a light brown background developed. Finally, the slides were immersed in deionized water several times. The TdT was replaced by PBS for the negative control.

For Ki67 staining, frozen slices were rinsed in PBS three times to remove the tissue-freezing matrix. Endogenous peroxidase activity was eliminated by incubation in $0.3 \%$ $\mathrm{H}_{2} \mathrm{O}_{2}$. The slides were treated with primary antibody of $\mathrm{Ki} 67$ (BD Biosciences, San Jose, CA) at a dilution of 1:100. The slides were then incubated with biotinylated secondary antibody (Santa Cruz Biotech, Santa Cruz, CA), washed, and
A

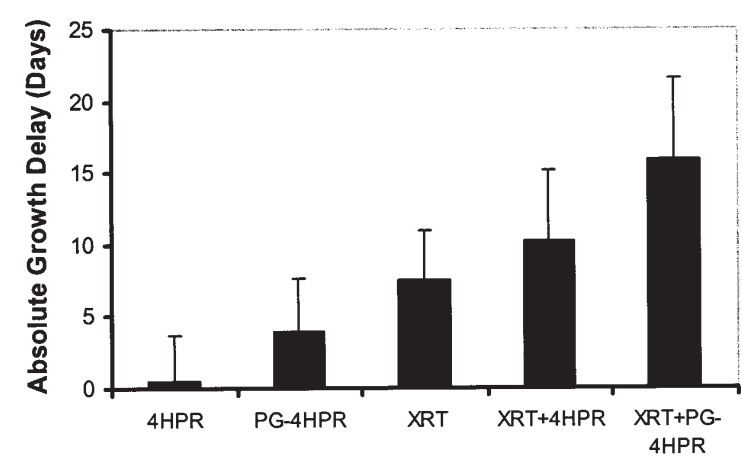

B

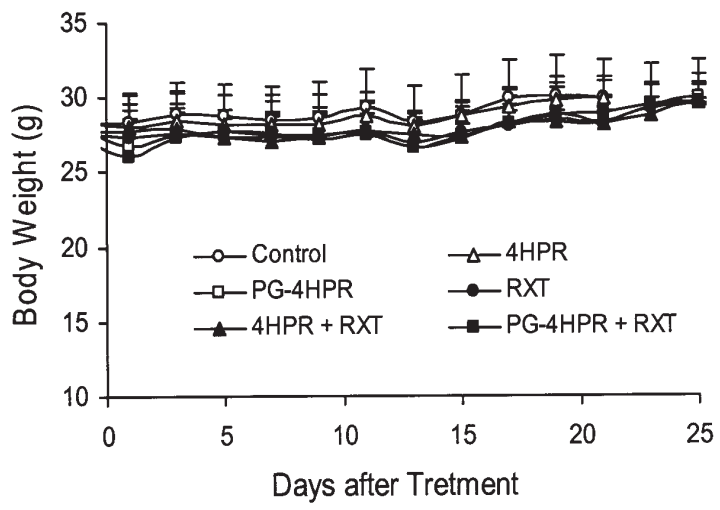

Figure 4. Antitumor activity of combined therapy in A549 xenografts. A: Therapy-induced tumor growth delay in days. Radiation was delivered as a single 10-Gy dose. 4-HPR or PG-4HPR was injected intravenously $24 \mathrm{~h}$ before local tumor irradiation at a dose of $30 \mathrm{mg}$ eq. $4 \mathrm{HPR} / \mathrm{kg}$ per injection. B: Body weight change as a function of time after the initiation of treatment. The data represent mean \pm standard error ( $n=6$ per group).

incubated with streptavidin-conjugated horseradish peroxidase. The slides were developed using ABC elite 3,3'-diaminobenzidine (Vector Lab, Inc., Burlingame, CA) and mounted in Permount (Fisher Scientific, Fair Lawn, NJ) for microscopic examination.

Table I. Effect of 4HPR and PG-4HPR on response of A549 tumors to radiation as measured by tumor growth delay.

\begin{tabular}{|c|c|c|c|c|}
\hline Treatment & $\begin{array}{l}\text { Days for tumors to grow from } \\
7.5 \text { to } 12.5 \mathrm{~mm}(\text { mean } \pm \mathrm{SE})\end{array}$ & $\begin{array}{l}\text { Absolute growth } \\
\text { delay }^{\mathrm{a}}\end{array}$ & $\begin{array}{l}\text { Normalized } \\
\text { growth delayb }\end{array}$ & $\begin{array}{c}\text { Enhancement } \\
\text { factor }^{c}(95 \% \mathrm{CI})\end{array}$ \\
\hline Nontreated control & $15.3 \pm 1.5$ & & & \\
\hline 4HPR & $15.8 \pm 3.2$ & $0.5 \pm 3.2$ & & \\
\hline PG-4HPR & $19.3 \pm 3.6^{\mathrm{d}}$ & $4.0 \pm 3.6$ & & \\
\hline XRT & $22.8 \pm 3.7$ & $7.5 \pm 3.5$ & & \\
\hline $\mathrm{XRT}+4 \mathrm{HPR}$ & $25.5 \pm 4.9$ & $10.2 \pm 4.9$ & $9.7 \pm 5.1$ & 1.3 \\
\hline XRT + PG-4HPR & $31.2 \pm 5.6^{\mathrm{e}}$ & $15.9 \pm 5.6$ & $11.9 \pm 5.6$ & 1.6 \\
\hline
\end{tabular}

${ }^{a}$ Absolute growth delay was defined as the time in days for tumors in treated groups to grow from 7.5 to 12.5 mm minus the time in days for tumors in the control group to grow to the same size. ${ }^{b}$ Normalized growth delay was defined as the time in days for tumors to grow from 7.5 to $12.5 \mathrm{~mm}$ in mice treated with the combined regimen minus the time to reach the same size in tumors treated with 4HPR or PG-4HPR alone. ${ }^{~}$ Enhancement factors were obtained by dividing normalized tumor growth delay by the absolute growth delay in mice treated with XRT alone. CI, confidence interval. ${ }^{\mathrm{d} C}$ Compared with that of control group: $0.01<\mathrm{p}<0.05$. ${ }^{\mathrm{e}}$ Compared with that of irradiation alone group: $0.01<\mathrm{p}<0.05$. 
The numbers of positive cells per $40 \mathrm{X}$ field of view were scored for both TUNEL and Ki67 using the method of Whitehead et al (20). Five fields of non-necrotic areas were selected randomly across each tumor section and the average number of positively stained cells per field was calculated.

Statistics. Differences in tumor diameter between the treatment groups were analyzed by one-way analysis of variance using Statistical Package for the Social Sciences (SPSS) software (SPSS Inc., Chicago, IL). The Mann-Whitney test was used to analyze differences between treatment groups in numbers of TUNEL- and Ki67-positive cells. A difference was considered statistically significant when the p-value was $<0.05$.

\section{Results}

Growth inhibition of A549 cells. Morphologically, cells treated with 4HPR or PG-4HPR became rounded and detached from the surface of culture plates, which indicates cell death. This effect was more pronounced at a longer incubation time, as reflected by a significant reduction in cell density compared to untreated control (Fig. 2). The effect of prolonged incubation on growth inhibition was more pronounced with PG-4HPR than with $4 \mathrm{HPR}$. The $50 \%$ inhibition concentration $\left(\mathrm{IC}_{50}\right)$ of PG-4HPR decreased from $>50 \mu \mathrm{M}$ to $9.75 \mu \mathrm{M}$ when exposure times increased from $24-120 \mathrm{~h}$. The $\mathrm{IC}_{50}$ of $4 \mathrm{HPR}$ was $25.8 \mu \mathrm{M}$ and $6.25 \mu \mathrm{M}$ after $24 \mathrm{~h}$ and $120 \mathrm{~h}$ exposure, respectively.

In vitro radiosensitivity. Treatment with 4HPR or PG-4HPR alone at a concentration of $10 \mu \mathrm{M}$ for $120 \mathrm{~h}$ reduced the number of A549 colonies to $65 \%$ and $68 \%$, respectively, that of untreated controls. The effect of combined treatments on colony formation was normalized to that of the corresponding drug used alone. Fig. 3 shows the survival curves for A549 cells grown without or with continuous supplementation of 4HPR and PG-4HPR and treated with varying doses of radiation. Cell survival decreased when 4HPR or PG-4HPR was used in combination with radiation. There was no difference in radiation response between $4 \mathrm{HPR}$ - and PG4HPR-treated cells, the two survival curves being nearly superimposable. Enhancement factors were calculated at the surviving fraction of 0.1 by dividing the radiation dose required to kill $90 \%$ of colonies with radiation alone with the dose required to kill $90 \%$ of colonies with radiation combined with 4HPR or PG-4HPR. The enhancement factors for 4HPR and PG-4HPR were 1.40 and 1.43 , respectively.

In vivo tumor response to radiation. Mice bearing A549 lung cancer xenografts were treated with 4HPR, PG-4HPR, radiation alone, 4HPR plus radiation, or PG-4HPR plus radiation. Tumor irradiation alone delayed tumor growth for $7.5 \pm 3.5$ days compared with untreated controls, whereas 4HPR alone at a single dose of $30 \mathrm{mg} / \mathrm{kg}$ did not delay tumor growth at all. PG-4HPR alone delayed tumor growth for $4.0 \pm 3.6$ days at a dose equivalent to $30 \mathrm{mg} / \mathrm{kg} 4 \mathrm{HPR}$. While the combination of $4 \mathrm{HPR}$ and XRT had a moderate inhibitory effect on tumor growth at the tested dose (10.2 \pm 4.9 days), the most effective tumor growth delay was achieved with a combination of PG-4HPR and XRT (15.9 \pm 5.6 day) (Table I and Fig. 4A). The radiation enhancement factors calculated for
$\mathbf{A}$

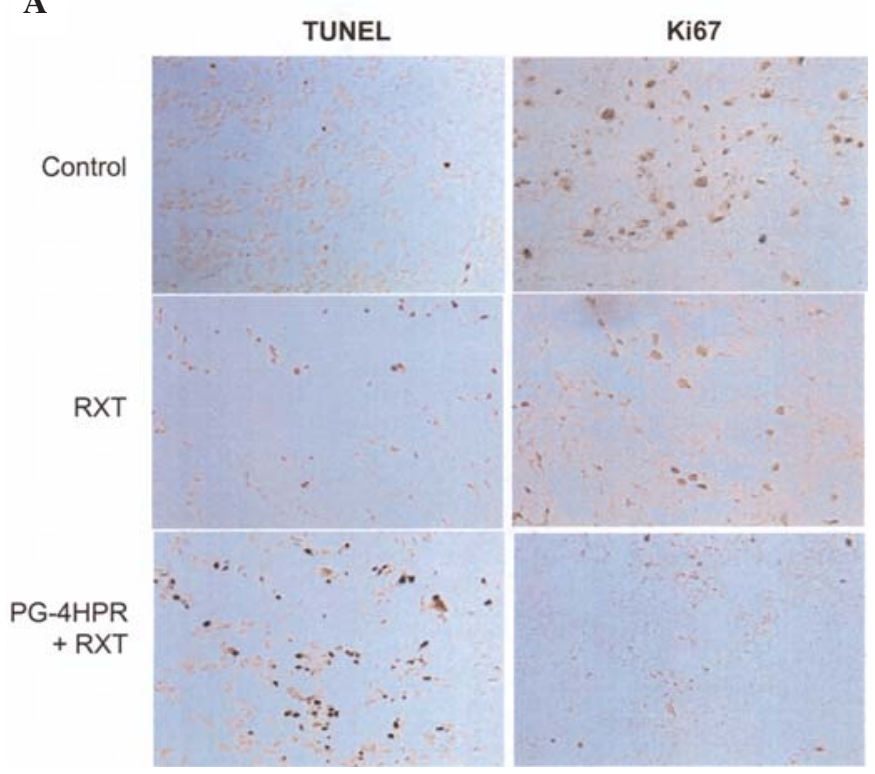

B
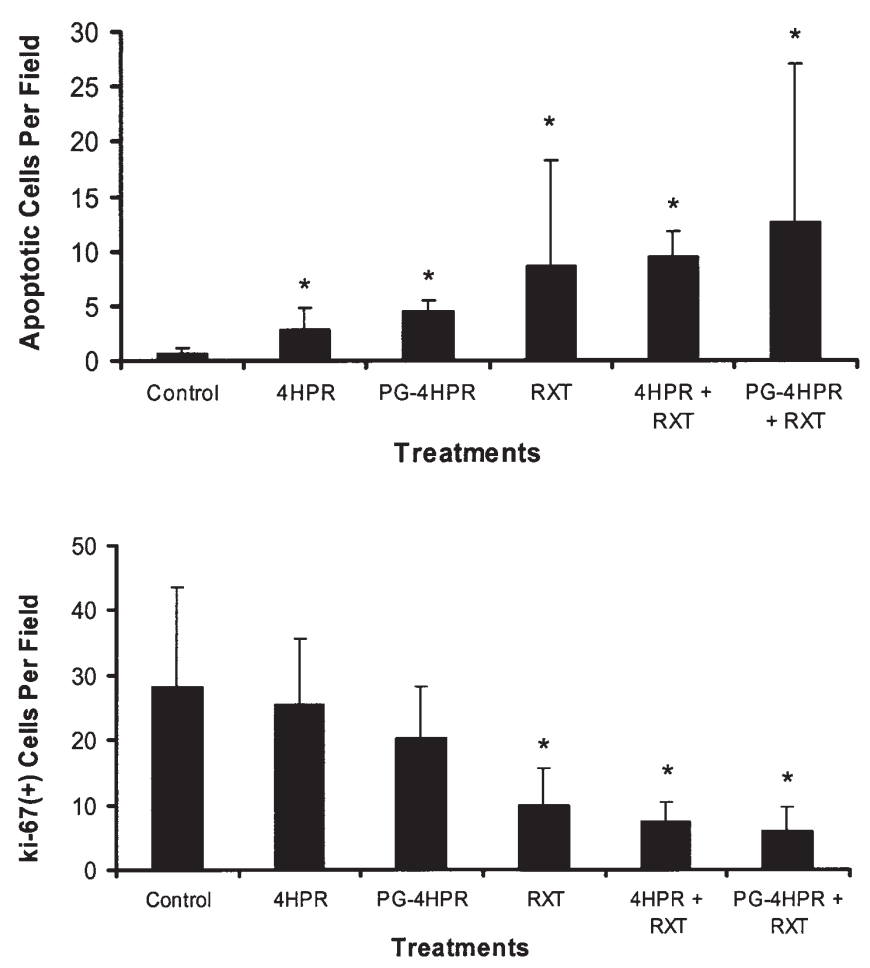

Figure 5. PG-4HPR enhanced in vivo apoptosis levels and decreased in vivo cell proliferation. Human lung A549 tumors from each treatment group were prepared and stained with TUNEL or Ki67 as described in Materials and methods. A: Representative microphotographs of TUNEL- and Ki67-stained A549 tumor sections for the control, radiation alone (10 Gy), and combined PG-4HPR (30 mg/kg)/radiation (10 Gy) groups. Original magnification: x10. B: Comparison of apoptotic cell counts (top panel) and proliferative cell counts (bottom panel) per field of stained slides for different treatments. The number of apoptotic cells and Ki67-positive cells per x40 fields of view were scored for each lung tumor. Data are presented as mean \pm standard deviation. ${ }^{*}$ p $<0.05$ compared to untreated control.

4HPR and PG-4HPR were 1.3 and 1.6, respectively. The PG$4 \mathrm{HPR} /$ radiation combination, but not the $4 \mathrm{HPR} /$ radiation combination, resulted in significantly better tumor growth 
control compared with radiation alone $(\mathrm{p}<0.05)$. Treatment with $4 \mathrm{HPR}$ or PG-4HPR at the injected dose of $30 \mathrm{mg}$ eq. $4 \mathrm{HPR} / \mathrm{kg}$ did not cause a significant loss in body weight (Fig. 4B).

Effect of combined treatments on apoptosis and tumor proliferation index. To assess the effects of combined therapies on tumor apoptosis and proliferation, sections from tumors subjected to various treatments were stained for TUNEL and Ki67. Fig. 5A shows representative microphotographs of stained sections from tumors treated with combined PG-4HPR/ radiation and radiation alone in comparison with untreated controls. Fig. 5B summarizes the numbers of cells that stained positive for TUNEL and Ki67. A trend of increasing apoptotic tumor cells and decreasing proliferating cells can be seen with the following sequence of treatment groups: untreated controls, 4HPR, PG-4HPR, 4HPR/radiation, and PG-4HPR/ radiation, although the difference between the combination treatment groups and the radiation alone group did not reach statistical significance. Treatments with PG-4HPR, radiation, 4HPR/radiation, and PG-4HPR/radiation significantly increased apoptotic cells and decreased Ki67-positive cells compared with untreated controls (Fig. 5B).

\section{Discussion}

The enthusiasm for 4HPR as a radiosensitizing agent comes from its ability to decrease cell growth and drive cells into apoptosis in many cell culture systems at dose levels that are nontoxic to normal tissues (13-16). Although 4HPR has previously been reported to influence cellular response to radiation (17-19), no study has been reported on the radiosensitizing effect of 4HPR in vivo. In the current study, we confirmed that 4HPR enhances the response of A549 lung tumor xenografts to radiotherapy. Moreover, we found that the polymeric conjugate PG-4HPR enhanced the radioresponse of lung cancer tumors to a greater extent than did free 4HPR (Fig. 4 and Table I). Combined PG-4HPR/XRT therapy, but not 4HPR/XRT, significantly inhibited tumor growth compared with XRT or PG-4HPR alone $(p<0.05)$. The enhancement factors obtained with combined PG-4HPR/XRT and 4HPR/ XRT were 1.6 and 1.3 , respectively, representing a $23 \%$ improvement in treatment efficacy when 4HPR was replaced with PG-4HPR. Our in vivo data also confirmed that the combination of PG-4HPR and XRT increased apoptotic responses and reduced cell proliferation compared to monotherapies (Fig. 5).

The increased radiosensitivity induced by PG-4HPR, as compared with free $4 \mathrm{HPR}$, in tumor xenografts may be explained by the EPR effect of macromolecules. We have previously shown that a conjugate of PG with paclitaxel, PGTXL, demonstrated a remarkably enhanced radiosensitizing effect when compared with that obtained with free paclitaxel (20). Enhanced uptake of PG-based polymeric contrast agent has also been demonstrated by magnetic resonance imaging (21). Our current findings are consistent with these earlier findings with PG-TXL in that enhanced radioresponse was achieved with the polymer-drug conjugates. With a mechanism of action similar to that of PG-TXL, PG-4HPR may enhance the radiosensitivity of A549 tumors to 4HPR by increasing the delivery of 4HPR to the tumor and prolonging drug exposure at the irradiated tumor site.

In contrast to its enhanced antitumor efficacy in vivo, PG-4HPR was found to be less potent than free 4HPR in inhibiting A549 cell growth in vitro at an equal dose level on the basis of $\mathrm{IC}_{50}$ values. However, the difference in $\mathrm{IC}_{50}$ values between PG-4HPR and 4HPR decreased when the drug exposure time increased from $24 \mathrm{~h}$ to $120 \mathrm{~h}$. This is because free 4HPR was gradually released from PG-4HPR; 60\% of 4HPR was released from PG-4HPR over a period of $120 \mathrm{~h}$ (data not shown). The clonogenic survival assay confirmed that, in vitro, $\mathrm{PG}-4 \mathrm{HPR}$ retained the radiosensitizing activity of 4HPR. Both agents were equally effective in potentiating the radiosensitivity of A549 cells, with enhancement factors of 1.40 and 1.43, respectively (Fig. 3). This is not surprising, considering that the cells in clonogenic assay were exposed to both agents for 18 days, which allowed most of the 4HPR in PG-4HPR to be released. These results support the hypothesis that the enhanced antitumor activity of PG-4HPR in combination with XRT in vivo results from the increased and prolonged delivery of 4HPR to the tumor, rather than an intrinsically different mechanism of action between PG$4 \mathrm{HPR}$ and free $4 \mathrm{HPR}$.

Histologic analysis confirmed that both 4HPR and PG4HPR significantly increased apoptosis in A549 tumors compared with untreated tumors. Moreover, combined PG4HPR/XRT therapy increased apoptotic responses and reduced cell proliferation compared to PG-4HPR or XRT used alone (Fig. 5). 4HPR-induced apoptosis involves several mechanisms, including the generation of reactive oxygen species, which is a classic radiation enhancer $(22,23)$. Therefore, one of the sources of the enhanced antitumor effects of combined therapy might be the generation of reactive oxygen species with 4HPR and PG-4HPR treatments.

$\mathrm{PG}$ is an attractive polymeric drug carrier owing to its biodegradability, biocompatibility, and ability to render waterinsoluble drugs water soluble when the drug molecules are covalently conjugated to the PG carrier $(24,25)$. PG has been used for the delivery of several chemotherapeutic agents. One such conjugate, PG-TXL, has successfully advanced into clinical phase III studies (25). In the current study, 4HPR was conjugated to partially cross-linked PG composed of linear PG of low molecular weight $\left(M_{n}=13,000\right)$ by linking them together through hydrolytically labile ester bonds (Fig. 1). It is thought that the partially cross-linked PG is degraded by two modes: enzymatic degradation of the PG backbone and hydrolytic degradation of the cross-linking ester linkage. The hydrolytic degradation would yield PG having a molecular weight lower than the renal clearance threshold (26). Further studies are needed to evaluate whether partially cross-linked PG could be cleared from the body at a faster rate than could a linear PG polymer of comparable molecular weight. PG4HPR was clearly well tolerated, as reflected by the minimal change in the body weight of mice treated with PG-4HPR at the indicated dose.

In conclusion, the combination of polymeric agents with radiotherapy is a novel approach to increase the therapeutic ratio of radiotherapy in patients with lung cancer. Further work is justified to examine the effect in more detail, as an increase in tumor radiosensitivity of $60 \%$ with an acceptable level of 
toxicity might translate into significantly higher rates of local tumor control with radiotherapy.

\section{Acknowledgements}

This study was supported in part by the John S. Dunn Foundation. The Centralized Histopathology Laboratory is supported by Cancer Center Support Grant CA16672 awarded by the National Cancer Institute, Department of Health and Human Services. We thank Dawn Chalaire for editorial assistance.

\section{References}

1. Bernier J and Schneider D: Cetuximab combined with radiotherapy: An alternative to chemoradiotherapy for patients with locally advanced squamous cell carcinomas of the head and neck? Eur J Cancer 43: 35-45, 2006.

2. Eifel PJ: Chemoradiotherapy in the treatment of cervical cancer. Semin Radiat Oncol 16: 177-185, 2006.

3. Lee JS, Komaki R, Fossella FV, Glisson BS, Hong WK and Cox JD: A pilot trial of hyperfractionated thoracic radiation therapy with concurrent cisplatin and oral etoposide for locally advanced inoperable non-small cell lung cancer: A 5-year follow-up report. Int J Radiat Oncol Biol Phys 42: 479-486, 1998.

4. Liao Z, Komaki R, Stevens C, Kelly J, Fossella F, Lee JS, Allen P and Cox JD: Twice daily irradiation increases locoregional control in patients with medically inoperable or surgically unresectable stage II-IIIb non-small cell lung cancer. Int J Radiat Oncol Biol Phys 53: 558-565, 2002.

5. Riesterer O, Tenzer A, Zingg D, Hofstetter B, Vuong V, Pruschy $\mathrm{M}$ and Bodis $\mathrm{S}$ : Novel radiosensitizers for locally advanced epithelial tumors: Inhibition of the pi3k/akt survival pathway in tumor cells and in tumor-associated endothelial cells as a novel treatment strategy? Int J Radiat Oncol Biol Phys 58: 361-368, 2004.

6. Maeda H, Wu J, Sawa T, Matsumura Y and Hori K: Tumor vascular permeability and the epr effect in macromolecular therapeutics: A review. J Control Release 65: 271-284, 2000.

7. Ke S, Milas L, Charnsangavej C, Wallace S and Li C: Potentiation of radioresponse by polymer-drug conjugates. J Control Release 74: 237-242, 2001.

8. Li C, Ke S, Wu QP, Tansey W, Hunter N, Buchmiller LM, Milas L, Charnsangavej $\mathrm{C}$ and Wallace S: Potentiation of ovarian oca-1 tumor radioresponse by poly (1-glutamic acid)paclitaxel conjugate. Int J Radiat Oncol Biol Phys 48: 1119-1126, 2000.

9. Li C, Ke S, Wu QP, Tansey W, Hunter N, Buchmiller LM, Milas L, Charnsangavej $\mathrm{C}$ and Wallace S: Tumor irradiation enhances the tumor-specific distribution of poly(1-glutamic acid)-conjugated paclitaxel and its antitumor efficacy. Clin Cancer Res 6: 2829-2834, 2000.

10. Milas L, Mason KA, Hunter N, Li C and Wallace S: Poly(1glutamic acid)-paclitaxel conjugate is a potent enhancer of tumor radiocurability. Int J Radiat Oncol Biol Phys 55: 707-712, 2003.
11. De Luca LM: Retinoids and their receptors in differentiation, embryogenesis, and neoplasia. FASEB J 5: 2924-2933, 1991.

12. Niles RM: Signaling pathways in retinoid chemoprevention and treatment of cancer. Mutat Res 555: 81-96, 2004.

13. Hong WK, Lippman SM, Itri LM, Karp DD, Lee JS, Byers RM, Schantz SP, Kramer AM, Lotan R, Peters LJ, et al: Prevention of second primary tumors with isotretinoin in squamous-cell carcinoma of the head and neck. N Engl J Med 323: 795-801, 1990.

14. Kalemkerian GP, Slusher R, Ramalingam S, Gadgeel S and Mabry M: Growth inhibition and induction of apoptosis by fenretinide in small cell lung cancer cell lines. J Natl Cancer Inst 87: 1674-1680, 1995 .

15. Ulukaya E and Wood EJ: Fenretinide and its relation to cancer. Cancer Treat Rev 25: 229-235, 1999.

16. Zou CP, Kurie JM, Lotan D, Zou CC, Hong WK and Lotan R: Higher potency of n-(4-hydroxyphenyl)retinamide than alltrans-retinoic acid in induction of apoptosis in non-small cell lung cancer cell lines. Clin Cancer Res 4: 1345-1355, 1998.

17. Benbrook D, Shen-Gunther J, Nunez E and Dynlacht J: Differential retinoic acid radiosensitization of cervical carcinoma cell lines. Clin Cancer Res 3: 939-945, 1997.

18. Scribner J, Dennis R and Benbrook DM: Retinoids enhance cisplatin-based chemoradiation in cervical cancer cells in vitro. Gynecol Oncol 85: 223-225, 2002.

19. Zou C, Wang L, Liebert M, Grossman HB, Lotan R and Wei Q: Combined effect of chemopreventive agent n-(4-hydroxyphenyl) retinamide (4-hpr) and gamma-radiation on bladder cancer cell lines. Int J Oncol 13: 1037-1041, 1998.

20. Whitehead CM, Earle KA, Fetter J, Xu S, Hartman T, Chan DC, Zhao TL, Piazza G, Klein-Szanto AJ, Pamukcu R, Alila H, Bunn PA Jr and Thompson WJ: Exisulind-induced apoptosis in a non-small cell lung cancer orthotopic lung tumor model augments docetaxel treatment and contributes to increased survival. Mol Cancer Ther 2: 479-488, 2003.

21. Wen X, Jackson EF, Price RE, Kim EE, Wu Q, Wallace S, Charnsangavej C, Gelovani JG and Li C: Synthesis and characterization of poly(l-glutamic acid) gadolinium chelate: A new biodegradable mri contrast agent. Bioconjug Chem 15: $1408-1415,2004$.

22. Oridate N, Suzuki S, Higuchi M, Mitchell M, Hong W and Lotan R: Involvement of reactive oxygen species in n-(4hydroxyphenyl)retinamide-induced apoptosis in cervical carcinoma cells. J Natl Cancer Inst 89: 1191-1198, 1997.

23. Sun SY, Li W, Yue P, Lippman SM, Hong WK and Lotan R: Mediation of n-(4-hydoxyphenyl)retinamide-induced apoptosis in human cancer cells by different mechanisms. Cancer Res 59: 2493-2498, 1999

24. Li C: Poly(1-glutamic acid)-anticancer drug conjugates. Adv Drug Deliv Rev 54: 695-713, 2002.

25. Singer JW, Shaffer S, Baker B, Bernareggi A, Stromatt S, Nienstedt D and Besman M: Paclitaxel poliglumex (xyotax; ct-2103): An intracellularly targeted taxane. Anticancer Drugs 16: 243-254, 2005.

26. Renkin EM, Watson PD, Sloop CH, Joyner WM and Curry FE: Transport pathways for fluid and large molecules in microvascular endothelium of the dog's paw. Microvasc Res 14: 205-214, 1977. 